\title{
Characterizing the Anticancer Treatment Trajectory and Pattern in Patients Receiving Chemotherapy for Cancer Using Harmonized Observational Databases: Retrospective Study
}

Hokyun Jeon ${ }^{1 *}$, MS; Seng Chan You ${ }^{2,3 *}$, MD, MS; Seok Yun Kang ${ }^{4}$, MD; Seung In Seo ${ }^{5}$, MD; Jeremy L Warner ${ }^{6}$, MD, MS; Rimma Belenkaya ${ }^{7}$, MA, MS; Rae Woong Park ${ }^{1,2}$, MD, PhD

\footnotetext{
${ }^{1}$ Department of Biomedical Sciences, Ajou University School of Medicine, Suwon, Gyeonggi-do, Republic of Korea

${ }^{2}$ Department of Biomedical Informatics, Ajou University School of Medicine, Suwon, Gyeonggi-do, Republic of Korea

${ }^{3}$ Department of Preventive Medicine, Yonsei University College of Medicine, Seoul, Republic of Korea

${ }^{4}$ Department of Hematology-Oncology, Ajou University School of Medicine, Suwon, Gyeonggi-do, Republic of Korea

${ }^{5}$ Department of Internal Medicine, Kangdong Sacred Heart Hospital, Hallym University College of Medicine, Seoul, Republic of Korea

${ }^{6}$ Division of Hematology and Oncology, Department of Medicine, Vanderbilt University Medical Center, Nashville, TN, United States

${ }^{7}$ Department of Health Informatics, Memorial Sloan Kettering Cancer Center, New York, NY, United States

*these authors contributed equally
}

\section{Corresponding Author:}

Rae Woong Park, MD, PhD

Department of Biomedical Informatics

Ajou University School of Medicine

Hong Jae Gwan, 5th Fl

164, World cup-ro, Yeongtong-gu

Suwon, Gyeonggi-do, 16499

Republic of Korea

Phone: 82312194471

Email: rwpark99@gmail.com

\section{Abstract}

Background: Accurate and rapid clinical decisions based on real-world evidence are essential for patients with cancer. However, the complexity of chemotherapy regimens for cancer impedes retrospective research that uses observational health databases.

Objective: The aim of this study is to compare the anticancer treatment trajectories and patterns of clinical events according to regimen type using the chemotherapy episodes determined by an algorithm.

Methods: We developed an algorithm to extract the regimen-level abstracted chemotherapy episodes from medication records in a conventional Observational Medical Outcomes Partnership (OMOP) common data model (CDM) database. The algorithm was validated on the Ajou University School Of Medicine (AUSOM) database by manual review of clinical notes. Using the algorithm, we extracted episodes of chemotherapy from patients in the EHR database and the claims database. We also developed an application software for visualizing the chemotherapy treatment patterns based on the treatment episodes in the OMOP-CDM database. Using this software, we generated the trends in the types of regimen used in the institutions, the patterns of the iterative chemotherapy use, and the trajectories of cancer treatment in two EHR-based OMOP-CDM databases. As a pilot study, the time of onset of chemotherapy-induced neutropenia according to regimen was measured using the AUSOM database. The anticancer treatment trajectories for patients with COVID-19 were also visualized based on the nationwide claims database.

Results: We generated 178,360 treatment episodes for patients with colorectal, breast, and lung cancer for 85 different regimens. The algorithm precisely identified the type of chemotherapy regimen in 400 patients (average positive predictive value $>98 \%$ ). The trends in the use of routine clinical chemotherapy regimens from 2008-2018 were identified for 8236 patients. For a total of 12 regimens (those administered to the largest proportion of patients), the number of repeated treatments was concordant with the protocols for standard chemotherapy regimens for certain cases. In addition, the anticancer treatment trajectories for 8315 patients were shown, including 62 patients with COVID-19. A comparative analysis of neutropenia showed that its onset in colorectal cancer regimens tended to cluster between days 9-15, whereas it tended to cluster between days 2-8 for certain regimens for breast cancer or lung cancer. 
Conclusions: We propose a method for generating chemotherapy episodes for introduction into the oncology extension module of the OMOP-CDM databases. These proof-of-concept studies demonstrated the usability, scalability, and interoperability of the proposed framework through a distributed research network.

(JMIR Med Inform 2021;9(4):e25035) doi: 10.2196/25035

\section{KEYWORDS}

antineoplastic combined chemotherapy protocols; electronic health record; cancer; pattern; chemotherapy; database; retrospective; algorithm; scalability; interoperability

\section{Introduction}

\section{Background}

In cancer research, real-world data, with the exception of cancer registries, have been relatively underused despite recent advances in information technology and the availability of data from electronic health records (EHRs) or administrative claims databases [1]. One of the major challenges to the active use of EHRs or claims databases in cancer research is the limited availability of clinically relevant structured data elements. The essential data elements for cancer research include records of anticancer treatment at the unit of the regimen, rather than the individual drugs used in the regimen. In order for researchers to obtain the data necessary for conducting a comparative study of the treatment regimens of patients with cancer, a labor-intensive preprocess is inevitable [2-4].

\section{Prior Work}

Previously, researchers developed algorithms to replace the manual endeavor of capturing the details of chemotherapy from medication histories [5-8]. Even though these studies carved paths toward the use of real-world evidence in cancer research, none of them focused on identifying and addressing organizational barriers. Due to heterogeneity in the structure and semantics of EHRs or claims databases across institutions and countries, none of these studies provided a scalable framework.

The Observational Health Data Sciences and Informatics (OHDSI) collaboration, which is a multistakeholder group organized for global collaboration studies, provides the Observational Medical Outcomes Partnership (OMOP) Common Data Model (CDM) to contribute to medical research on harmonized observational databases [9]. The oncology work group in the OHDSI community has proposed an oncology module to facilitate the difficult task of collecting oncology data [10]. The oncology module has a structure for populating chemotherapy episodes and a vocabulary for hematology/oncology [11]. However, a generalizable method of populating chemotherapy episodes has not been proposed, and cases involving the use of data schema are scarce.

\section{Objectives}

The main objective of this study was the seamless introduction of the oncology extension into OMOP-CDM by developing an algorithm to automatically identify regimen-level chemotherapy episodes among patients with cancer. To conduct proof-of-concept studies of the availability of the generated chemotherapy episodes, the treatment patterns and trajectories of patients with cancer are presented by additional software. We also identified differences in the onset time and incidence of neutropenia events in patients according to different routine regimens.

\section{Methods}

\section{Study Design}

This study was composed of two main processes: (1) the development of an algorithm to identify anticancer treatment episodes from the OMOP-CDM database and (2) the analysis of the trends and trajectories in cancer treatment or clinical events based on the algorithm-derived episode records using the visualization software. Furthermore, we performed a pilot study to identify the time of neutropenia onset across various chemotherapy regimens, to validate the scalability of the algorithm. All methods were independently applied to each database and data were collected exclusively as graphical summaries.

\section{Data Sources}

We conducted this study using two EHRs of Korean tertiary hospitals and a nationwide claims database from South Korea. The Ajou University School of Medicine (AUSOM) database includes the medical records of 3.14 million patients collected from 1994-2018 [12]. The Kangdong Sacred Heart Hospital $(\mathrm{KDH})$ database includes the medical records of 1.68 million patients collected from 1986-2018. The Health Insurance Review and Assessment Service (HIRA) COVID-19 data set is a nationwide administrative claims database that provides information on reimbursed insurance claims from 2017-2020 for 7590 patients with COVID-19 in South Korea [13]. Each data source was standardized into the OMOP-CDM database (version 5.3). According to the medical history and diagnosis, we identified patients with lung, breast, and colorectal cancer from the EHR databases. From the HIRA COVID-19 data set, we selected patients with COVID-19 and any malignant neoplasm disease as the targets of the descriptive analysis.

\section{Algorithm Development}

\section{Workflow of the Chemotherapy Regimen Extraction}

Figure 1 shows the entire process used for generating chemotherapy episodes from medication records in harmonized databases. HemOnc is a standard vocabulary adopted by the OMOP-CDM for anticancer agents and chemotherapy regimens derived from the homonymous wiki page, including freely available medical sources for regimens and general information [14]. To convey the semantic regimen protocols in HemOnc to the programmatic algorithm, the section of the protocols 
detailing the regimen was collected and converted to parameterized values that list the component drugs as well as appropriate time criteria that reflect the drug schedule. Hence, we developed the Hierarchical Description for Administration of Chemotherapy (HDAC), which is a machine-readable JavaScript Object Notation (JSON) snippet containing the parameterized information of HemOnc. The HDAC contains the variables for the constituent drug of the regimen and the

Figure 1. Schematic workflow of the chemotherapy episode extraction. A total of 1506 regimen protocols from HemOnc (a web-based open-source database of cancer chemotherapy regimens) were parameterized as JSON structured data, which is termed HDAC. The JSON file and single drug exposure records in the OMOP-CDM database were instantiated as input data for an algorithm. The TRACER identified chemotherapy episodes by leveraging the parameters from HDAC. The chemotherapy episodes were curated in the episode table, which is an oncology module in the OMOP-CDM. Bev: bevacizumab; CDM: Common Data Model; FOLFIRI: fluorouracil, leucovorin, and irinotecan; FOLFOX: fluorouracil, leucovorin, and oxaliplatin; HDAC: Hierarchical Description for Administration of Chemotherapy; JSON: JavaScript Object Notation; OMOP: Observational Medical Outcomes Partnership; TRACER: Tool for Regimen-level Abstraction of Chemotherapy Episode Record.

\begin{tabular}{cccc}
\hline \multicolumn{3}{c}{ Drug exposure } \\
\hline Person & Drug & Drug_start_datetime & Drug_end_datetime \\
\hline 1 & Fluorouracil & $2019-09-08$ & $2019-09-08$ \\
1 & Alprazolam & $2019-09-08$ & $2019-09-08$ \\
1 & Leucovorin & $2019-09-09$ & $2019-09-09$ \\
1 & Sodium Chloride & $2019-09-09$ & $2019-09-09$ \\
1 & Oxaliplatin & $2019-09-09$ & $2019-09-09$ \\
\hline
\end{tabular}

time-range value parameters for the temporal window of the medication schedule.

We developed a Tool for Regimen-level Abstraction of Chemotherapy Episode Records (TRACER) to populate the regimen-level abstracted chemotherapy episodes (Figure 2). The TRACER generated the episodes by leveraging the parameter values of drug conditions or temporal window in HDAC. The chemotherapy episodes included the type of regimen and the number of treatment cycles.

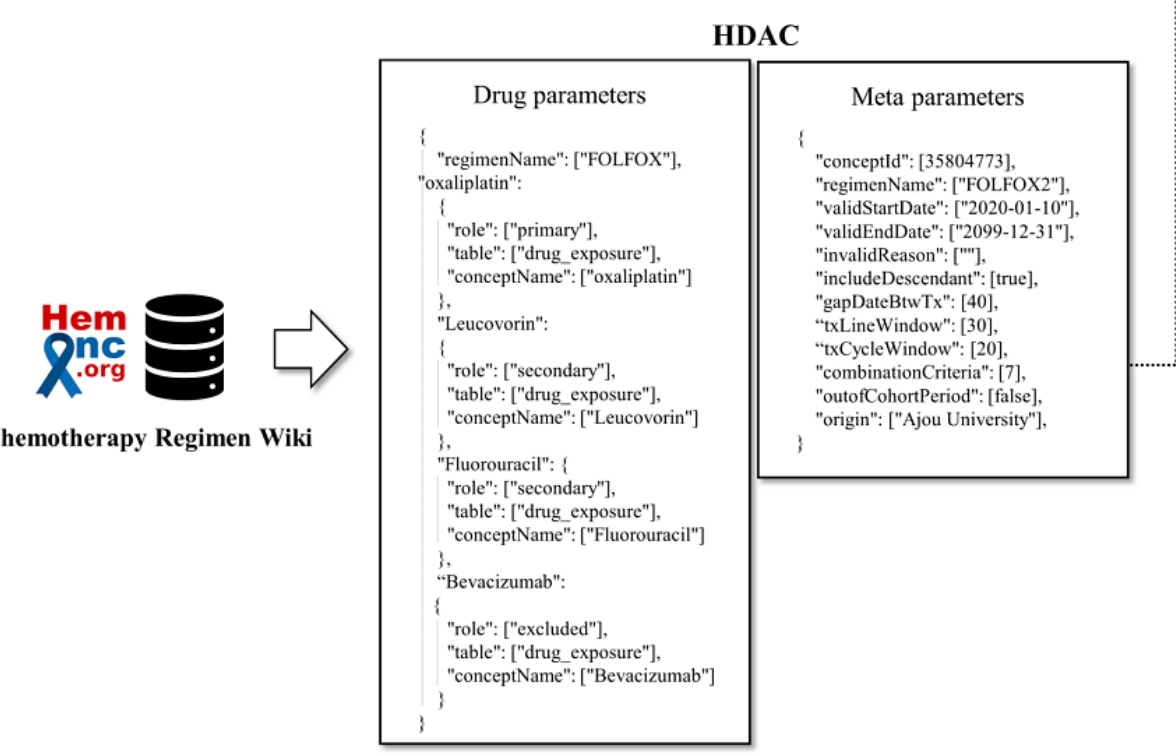

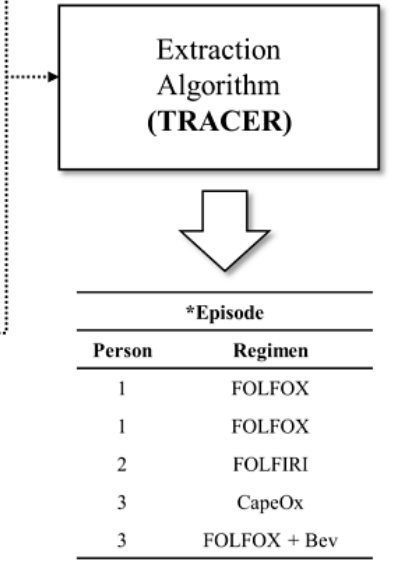

*Episode includes chemotherapy records : (1) treatment line and (2) each cycle level 
Figure 2. Schematic depiction of algorithm rules in a tool for regimen-level abstraction of chemotherapy episode records. FOLFOX: fluorouracil, leucovorin, and oxaliplatin.

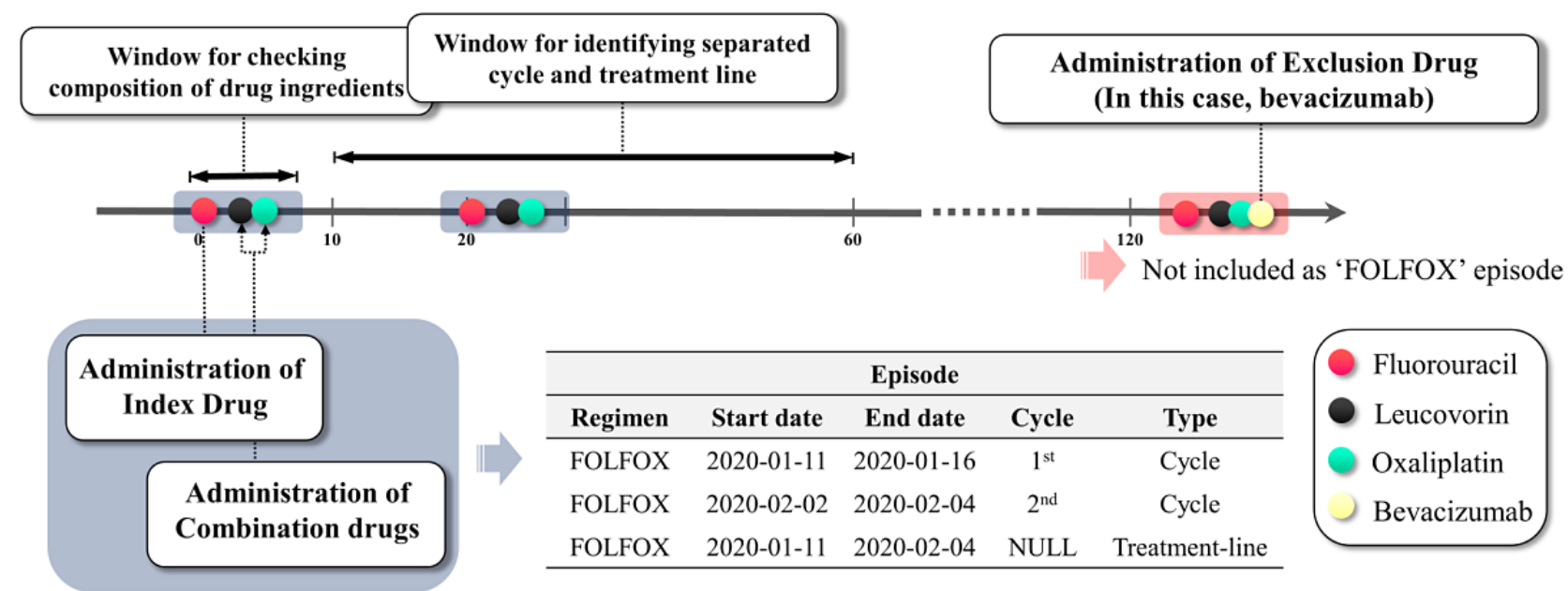

\section{Instantiation of Chemotherapy Regimen Descriptions}

The HDAC includes standardized parameters to feed the specifications of regimen protocols into the algorithm. The variables in HDAC are categorized into two types: parameters for drug composition (drug parameters) and meta parameters.

The drug parameter includes the identifiers of drugs (OMOP concept IDs), which link a specific regimen with its respective role. Each drug parameter is granted a role as an index drug, combination drug, or exclusion drug. The index drug in the HDAC is a constituent drug that can be used to identify the first day (day 1) of treatment. A combination drug in the HDAC is a constituent drug (other than the index drug) of the regimen. An exclusion drug is one whose appearance would indicate another regimen. For example, oxaliplatin is the index drug in the fluorouracil, leucovorin, and oxaliplatin (FOLFOX) regimen. Leucovorin and fluorouracil are combination drugs. In this example, bevacizumab is considered as an exclusion drug to distinguish the FOLFOX regimen from the FOLFOX-bevacizumab regimen (Figure 2).

The meta parameter includes the metadata of the HDAC document (eg, origin, valid date, or invalid reason for document) to determine the modifications and define the window range to be adjusted to the algorithm rule. The window identifies a unit of drug records that determines whether the medication record is a part of a particular regimen or distinguishes a boundary for a distinct treatment cycle. The HDAC also stipulates the window for episodes to distinguish the separated treatment line. The concept ID (encoded in the HemOnc vocabulary) of the drug regimen is a primary key for each HDAC snippet. Based on the chemotherapy indications in the HemOnc web database, a total of 1506 indications for chemotherapy protocols were reviewed by an expert and instantiated into the HDAC.

\section{Definitions of the Algorithm}

The TRACER sequentially extracts the episodes of regimens included in a list of user settings. The algorithm identifies each treatment cycle episode record and treatment line episode of regimens with the defined rules and parameters in a step-by-step manner (Figure 3 ). The algorithm consists of the following four steps:

1. Day 1 of the respective treatment cycle (index date) is identified based on the dispense date of the index drug. Each index date is flagged as a datum point for checking the use of other drug ingredients to identify a specific regimen.

2. The prescriptions of the combination drug or exclusion drug are investigated within the period of the predefined window for the cycle in HDAC. If the index drug and all combination drugs were given and none of the exclusion drugs were prescribed in this period, the records of the index drug and combination drug are regarded as a constituent component for a targeting regimen. These records are abstracted as a regimen episode record.

3. The start date of each episode is derived from the start date of the instance of index drug utilization, and the end date of the episode record is derived from the end date of the last index or combination drug utilization. The generated episodes are curated in the episode table of the OMOP-CDM oncology module.

4. The episodes are numbered sequentially in chronological order, provided the interval between the start dates of each episode does not exceed the predefined window in the HDAC. The episode records are collapsed as an identical episode when the interval exceeds the cycle window. The window for distinguishing the treatment line is also defined in the HDAC. The TRACER distinguishes the different treatment lines by changes in regimen type or by episodes beyond the window for the treatment line based on the start date of the previous episode. 
Figure 3. Definition of chemotherapy regimen episode extraction algorithm. FOLFIRI: fluorouracil, leucovorin, and irinotecan; FOLFOX: fluorouracil, leucovorin, and oxaliplatin; HDAC: Hierarchical Description for Administration of Chemotherapy.

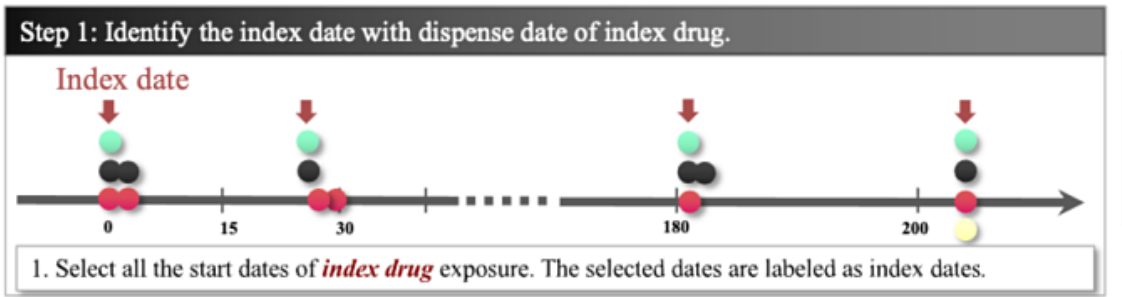

Step 2: Investigate the drug conditions around the index date.

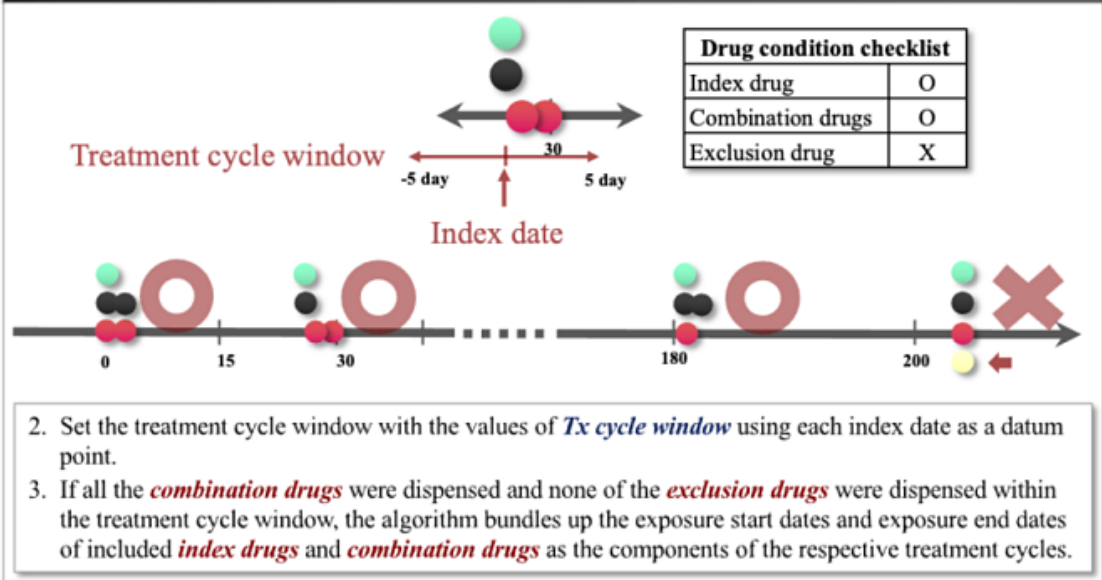

Step 3: Abstract the drug records as chemotherapy cycle episode records

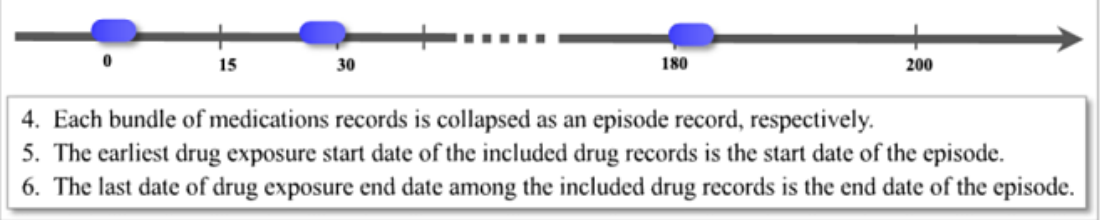

Step 4: Distinct treatment cycles as separate treatment lines

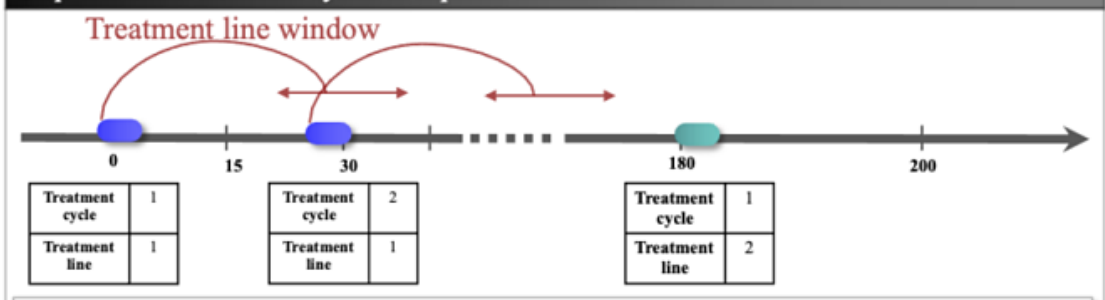

7. The treatment cycle and line of the first episode are assigned to 1 .

8. Set the treatment line window with the values of $T x$ line window using the start date of the first episode as a datum point.

9. If the start date of the next episode record in chronological order precedes the treatment line window, the treatment cycle of the episode record is collapsed with the previous episode record.

10.If the start date of the next episode record in chronological order is included within the range of the treatment line window, the previous treatment cycle number +1 is assigned to the treatment cycle.

11.If the start date of the next episode record in chronological order follows the treatment line window, the previous treatment line number +1 is assigned to the treatment line and number 1 is assigned to the treatment cycle.

12.Repeat steps 8-11 using the previously numbered episode as a new datum point until the last episode record is numbered.

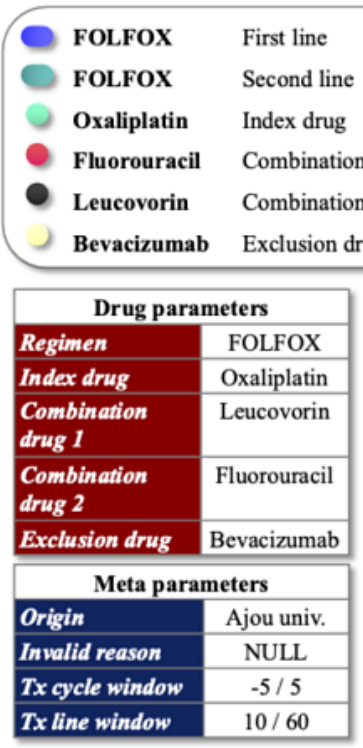

\section{Algorithm Validation}

We reviewed the discharge and progress notes of patients to validate the accuracy of the proposed algorithm. The following regimens were validated: (1) fluorouracil and folinic acid (FULV), (2) FOLFOX, (3) fluorouracil, leucovorin, and irinotecan (FOLFIRI), and (4) capecitabine monotherapy. Among patients with records of algorithm-derived episodes on target regimens, 100 patients were randomly selected for each type of regimen. For this population, we examined the episode records and compared them to clinical notes.

\section{Characterizing the Treatment Patterns and Trajectories}

We developed visualization applications for characterizing the treatment patterns for patients with cancer treated with 
chemotherapy. Using the tool, we present the relative proportions of the use of the regimens across all chemotherapy treatments from 2008-2018. The distributions of the iterated number of treatment cycles for each patient according to regimen type were portrayed as a heat map with color saturation varying according to the number of patients. The anticancer treatment trajectories were also illustrated for patients who received routine anticancer treatment according to the type of cancer. The trajectories of patients with COVID-19 and cancer were added, to validate the scalability of the tools. The descriptive results of EHR databases included the eight most prevalent regimen types. For the AUSOM database, we added hormone therapies for breast cancer and targeted therapies or immunotherapy for lung cancer in the descriptive analysis.

\section{Timing of Neutropenia Onset Analysis}

We also conducted a pilot study that investigated the timing of chemotherapy-induced (febrile) neutropenia (CIN/FN) events from the first chemotherapy episode. Neutropenia is a common adverse event of myelosuppressive chemotherapy. In compliance with National Cancer Institute Common Terminology Criteria for Adverse Events (CTCAE; version 5.0) CIN grade 4 (absolute neutrophil count $\left.[\mathrm{ANC}]<0.5 \times 10^{9} / \mathrm{L}\right)$ was used to identify severe CIN events. FN events were identified as ANC of $<1.0$ $\times 10^{9} / \mathrm{L}$ with a diagnosis of fever or infection, or any use of granulocyte colony-stimulating factor prophylaxis. A plot showing each neutropenia event as a dot by date of onset was displayed, and in the same plot, a violin plot showed the trends in date of neutropenia onset. To identify the onset time of the neutropenic event, the gap between the date of the first chemotherapy treatment and the date of the first CIN/FN onset was calculated for each patient. As the overall measurement schedule was weekly, the onset dates of neutropenia were categorized in 7-day segments to show the trends of onset dates rather than definite dates. To clarify the effect of a single drug regimen on neutropenia onset, the chemotherapies are limited to the first-line treatment and only the CIN/FN events within 30 days of initiation of chemotherapy were considered. On the day of chemotherapy, the ANC level might be temporarily lowered; therefore, a CIN/FN event during chemotherapy administration was ignored. We also depicted the incidence of CIN/FN events in each cycle by regimen type. The four regimens with the highest frequency of CIN/FN events by type of cancer were included for the incidence plot.

\section{Statistical Analysis}

The descriptive analysis was conducted using chemotherapy episodes that were derived from the algorithm. To validate the algorithm, we calculated the proportion of patients who had episodes with identical regimen types as described in clinical notes. The mean absolute error (ie, the mean of the absolute values for the differences between the estimated number of cycles and the actual records in the clinical notes) and the root mean square error (ie, the square root of the mean of the differences between the estimated number of cycles and the actual records in the clinical notes) were also calculated. The overall system was built using $\mathrm{R}$ (version 3.5.2; R Foundation for Statistical Computing). The source codes for the algorithm and visualization software have been uploaded to GitHub [15].

\section{Ethics Statement}

This study was approved by the institutional review board of Ajou University Hospital of the Republic of Korea (approval number: AJIRB-MED-OBS-20-092) and Kangdong Sacred Heart Hospital of the Republic of Korea (approval number: 2017-03-003). The institutional review board number for the use of HIRA data was AJIRB-MED-EXP-20-087.

\section{Results}

\section{Population Characteristics}

The TRACER generated a total of 178,360 chemotherapy episodes from the AUSOM database. The episodes consisted of 12 regimen types for colorectal cancer, 24 types for breast cancer (including 6 types of hormone therapy regimen), and 19 types for lung cancer (including 8 types of targeted therapy regimen). The number of patients who were treated with the respective regimens are listed in Multimedia Appendix 1. The characteristics of patients in the AUSOM database are listed (Table 1). Among the 10,353 colorectal, 9546 breast, and 12,671 lung cancer cases, 3151 (30.4\%), 5568 (58.3\%), and 1593 $(12.5 \%)$ patients had records of a treatment episode, respectively. The number of patients treated with chemotherapy increased over the years. A total of 69,353 chemotherapy episodes were extracted from the KDH database. The KDH database included 2758 patients with colorectal cancer, 564 (20.4\%) of whom had chemotherapy episodes. Among the patients with breast cancer $(n=6420)$ and lung cancer $(n=2663)$ in the $\mathrm{KDH}$ database, chemotherapy episodes were identified for $1075(16.7 \%)$ and $642(24.1 \%)$ patients, respectively. The HIRA COVID-19 data set mostly consisted of female patients (60\%) [13]. Among 7590 patients with COVID-19, we identified $382(5 \%)$ patients with a primary malignant neoplastic disease. 
Table 1. Characteristics of patients with colorectal, breast, and lung cancer in the Ajou University School of Medicine database.

\begin{tabular}{|c|c|c|c|}
\hline \multirow[t]{2}{*}{ Characteristics of the patients } & \multicolumn{3}{|l|}{ Patients by type of cancer } \\
\hline & Colorectal cancer $(\mathrm{N}=10,353)$ & Breast cancer $(\mathrm{N}=9546)$ & Lung cancer $(\mathrm{N}=12,671)$ \\
\hline Age at index (years), mean (SD) & $62(13.2)$ & $50(11.4)$ & $64(12.7)$ \\
\hline \multicolumn{4}{|l|}{ Sex, n (\%) } \\
\hline Male & $6116(59.1)$ & $62(0.7)$ & $9166(72.3)$ \\
\hline Female & $4237(40.9)$ & $9484(99.3)$ & $3505(27.7)$ \\
\hline \multicolumn{4}{|c|}{ Number of patients who received chemotherapy by year range, $n(\%)$} \\
\hline 1999-2002 & $265(2.6)$ & $646(6.8)$ & $524(4.1)$ \\
\hline 2003-2006 & $516(5.0)$ & $829(8.7)$ & $617(4.9)$ \\
\hline $2007-2010$ & $672(6.5)$ & $965(10.1)$ & $738(5.8)$ \\
\hline $2011-2014$ & $852(8.2)$ & $1373(14.4)$ & $775(6.1)$ \\
\hline $2015-2018$ & $912(8.8)$ & $2111(22.1)$ & $1127(8.9)$ \\
\hline Number of patients who underwent surgery, n (\%) & $3760(36.3)$ & $5541(58.0)$ & $1776(14.0)$ \\
\hline Baseline absolute neutrophil count $/ \mu \mathrm{L}$, mean (SD) & $5582(4403)$ & $3750(3199)$ & $6517(5283)$ \\
\hline \multicolumn{4}{|c|}{ Number of patients who received chemotherapy, $n(\%)$} \\
\hline First-line treatment & $3151(30.4)$ & $5568(58.3)$ & $1593(12.5)$ \\
\hline Second-line treatment & $1212(11.7)$ & 4739 (49.6) & $888(7.0)$ \\
\hline Third-line treatment & $506(4.8)$ & 4005 (41.9) & $521(4.1)$ \\
\hline Fourth-line treatment & $234(2.2)$ & $3573(37.4)$ & $336(2.6)$ \\
\hline
\end{tabular}

\section{Validation of the Accuracy of TRACER}

Table 2 shows the values obtained for the accuracy of the algorithm-derived episode records compared to the clinical notes. The positive predictive value for the type of regimen was over $95 \%$ for the FULV regimen, and the chemotherapy type was estimated precisely for the entire episode for FOLFOX, FOLFIRI, and capecitabine monotherapy. The number of treatment cycles was correctly inferred in an average of $85.6 \%$ of episode records for validated regimens. The mean difference between the number of cycles in the episode records and the description in the clinical notes was less than one cycle.

Table 2. Validation of chemotherapy episodes compared to chart review.

\begin{tabular}{llllll}
\hline Treatment regimen & Without information, $\mathrm{n}$ & $\begin{array}{l}\text { Positive predictive value } \\
\text { regimen type, } \mathrm{n} / \mathrm{N}(\%)\end{array}$ & $\begin{array}{l}\text { Accuracy of treatment cycle } \\
\text { number, \% }\end{array}$ & $\begin{array}{l}\text { Mean absolute } \\
\text { error }\end{array}$ & $\begin{array}{l}\text { Root mean } \\
\text { square error }\end{array}$ \\
\hline FULV $^{\mathrm{d}}$ & 30 & $67 / 70(95)$ & 94 & 0.1 & 0.4 \\
FOLFOX & & $92 / 92(100)$ & 87 & 0.3 & 0.6 \\
$\begin{array}{l}\text { FOLFIRI } \\
\text { Capecitabine }\end{array}$ & 21 & $79 / 79(100)$ & 89 & 73 & 0.4 \\
monotherapy
\end{tabular}

${ }^{a}$ For each regimen, 100 cases were randomly sampled and reviewed. The information for chemotherapy was not available in the discharge summary in 30, 8, 21, and 65 cases with FULV, FOLFOX, FOLFIRI, and capecitabine monotherapy, respectively.

${ }^{\mathrm{b}}$ Positive predictive value for the matched cases for the type of regimen in the manual comparison of generated episode records with the contents of the clinical notes.

${ }^{\mathrm{c}}$ The percentage of matched cases for the number of treatment cycles in the manual comparison of generated episode records with the contents of the clinical notes.

${ }^{\mathrm{d}}$ FULV: fluorouracil and leucovorin.

${ }^{\mathrm{e}}$ FOLFOX: fluorouracil, leucovorin, and oxaliplatin.

${ }^{f}$ FOLFIRI: fluorouracil, leucovorin, and irinotecan.

\section{Patterns of Chemotherapy Treatment}

The trends in chemotherapy regimen used in the AUSOM database are shown in Multimedia Appendix 2. The number of chemotherapy regimens-including targeted anticancer agents (eg, osimertinib, ceritinib, and crizotinib) and immunotherapies (eg, nivolumab and pembrolizumab) - for lung cancer increased since 2016. On average, $31.2 \%$ of patients received the 
FOLFOX regimen throughout the years, followed by FULV, which was administered to $20.3 \%$ of patients. As of 2012, the proportion of patients receiving chemotherapy with targeted anticancer drugs (eg, bevacizumab or cetuximab) had increased gradually. Tamoxifen use also increased gradually among patients with breast cancer. Multimedia Appendix 3 shows the trends in chemotherapy regimen use in the KDH database. The most frequently used regimen for colorectal cancer in the $\mathrm{KDH}$ database was also FOLFOX (27.3\% on average), followed by FULV, with an average of $23 \%$. The use of regimens including targeted anticancer drugs for colorectal cancer in the $\mathrm{KDH}$ database increased since 2013. From 2013-2016, gefitinib monotherapy was the most frequently used regimen for lung cancer, which was similar to the trends of the AUSOM database. Among the patients with breast cancer in the KDH database, the use of trastuzumab increased sharply since 2014.

The distribution of patients in both EHR databases over the number of iterated chemotherapy cycles is depicted as a heat map. In the heat map of the AUSOM database, the most prevalent number of repeated cycles in the FULV, FOLFOX, and capecitabine and oxaliplatin (CapeOx) regimens for colorectal cancer was consistent with the recommendations of the HemOnc regimen protocol for the adjuvant setting $(6,12$, and 8 cycles, respectively; Figure 4$)$. In addition, the most prevalent number of treatment iterations for paclitaxel monotherapy, doxorubicin monotherapy, and cyclophosphamide and doxorubicin (AC) targeting breast cancer was also consistent with the general recommendations (4 cycles in certain cases). "Cisplatin and vinorelbine" and "cisplatin and pemetrexed" for lung cancer were also consistent with the recommendations (4 cycles in certain cases). In the $\mathrm{KDH}$ database, the number of patients with colorectal cancer treated with 12 cycles made up the largest proportion among the patients who received the FOLFOX regimen (Multimedia Appendix 4). A total of four regimens-fluorouracil, epirubicin, and cyclophosphamide (FEC); fluorouracil, doxorubicin, and cyclophosphamide (FAC); cyclophosphamide, methotrexate, and fluorouracil (CMF); and Taxotere-for breast cancer were mostly repeated 6 times, and each of the regimen protocols in HemOnc included 6 cycles in certain cases. 
Figure 4. Heat map of patient distribution for cycle iteration by regimen type in the Ajou University School of Medicine database. The number of patients with (A) colorectal cancer, (B) breast cancer, and (C) lung cancer is shown; treatment iteration count is represented by color saturation, with a darker shade representing a higher number of patients. Cells with <10 patients are not reported. AC: doxorubicin and cyclophosphamide; CapeOx: capecitabine and oxaliplatin; FAC: fluorouracil, doxorubicin, and cyclophosphamide; FOLFIRI: fluorouracil, leucovorin, and irinotecan; FOLFOX: fluorouracil, leucovorin, and oxaliplatin.

\section{A}

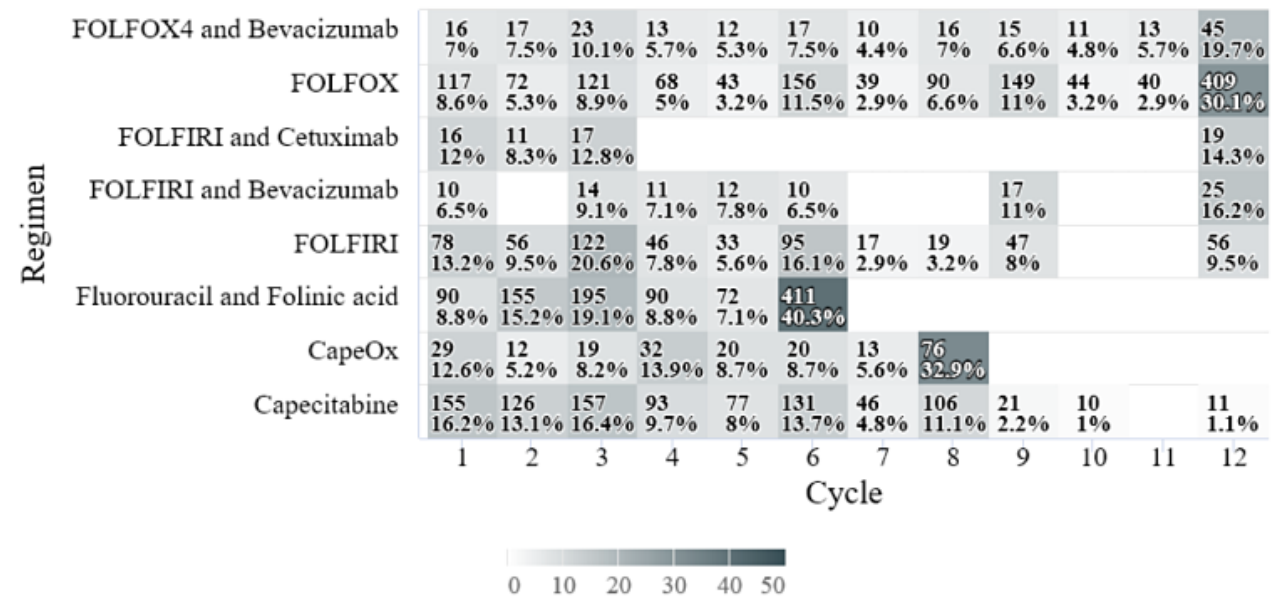

B
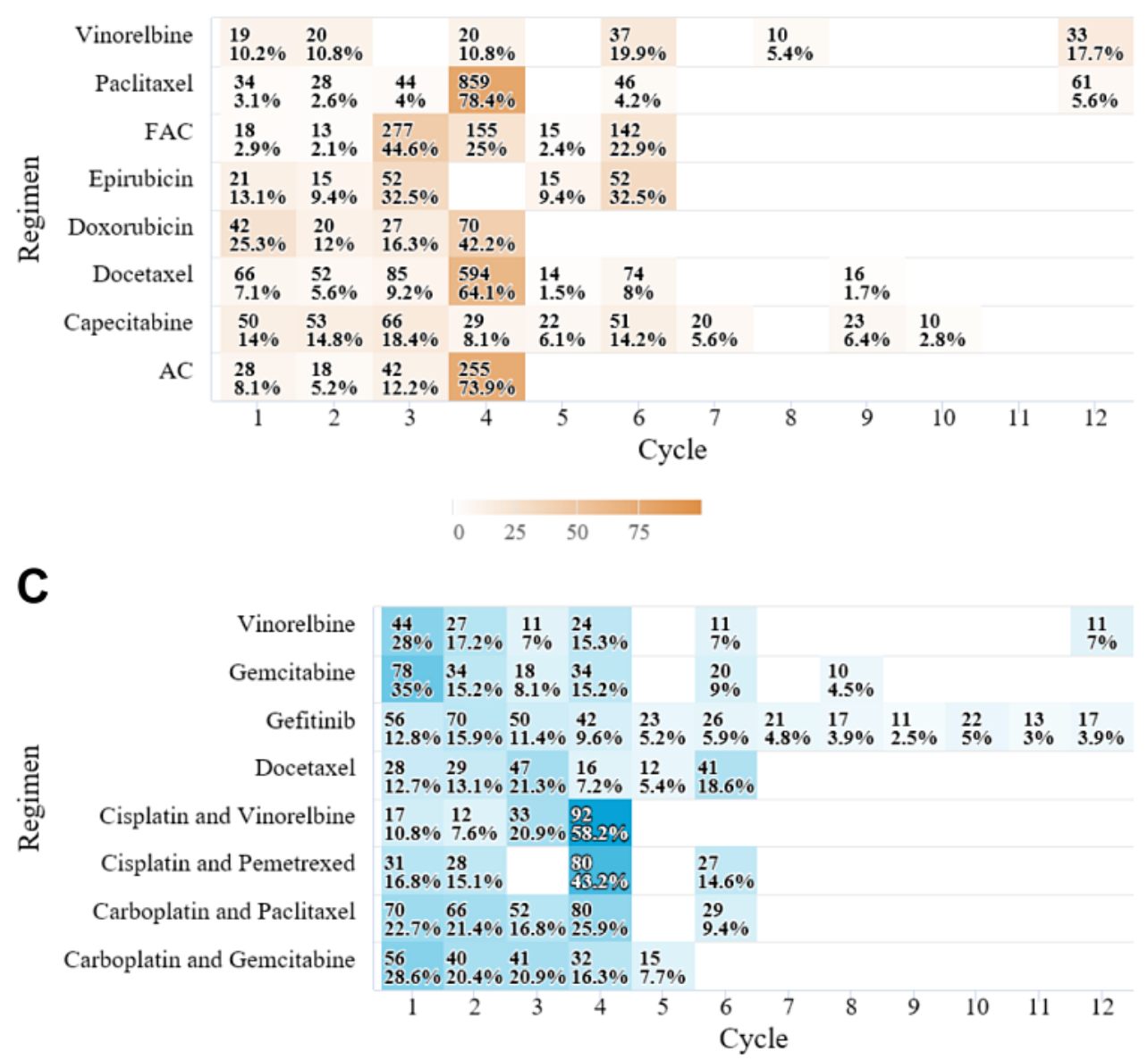

$0 \quad 20 \quad 40 \quad 60$

\section{Trajectory of Cancer Treatment}

Figure 5 shows the treatment trajectories of patients with lung cancer in the AUSOM database. Among a total of 1120 patients, lung excision-radiation therapy-cisplatin and vinorelbine $(\mathrm{n}=63$, $5.6 \%$ ) was the most prevalent trajectory. The treatment trajectories of patients with colorectal or breast cancer were displayed, regardless of which first-line treatment was used (Multimedia Appendix 5). Among the treatment trajectories of patients with colorectal cancer or breast cancer that included at least three treatments, colectomy-FOLFOX-FOLFIRI $(n=78$, $4 \%)$ and mastectomy-AC-paclitaxel monotherapy $(n=236,5 \%)$ 
were the trajectories with the highest proportions, respectively. described in Multimedia Appendix 6. The 10 most frequent trajectories according to cancer type are

Figure 5. Anticancer treatment trajectories of patients with lung cancer in the Ajou University School of Medicine database. The treatment trajectories of patients with lung cancer were classified according to the type of first-line treatment: (A) surgery, (B) chemotherapy or chemotherapy with radiation, and $(C)$ radiation therapy. The height of each node represents the population of patients in the corresponding treatment line or therapy. The number of patients who progressed to the next line of treatment is illustrated using gray lines. The chemotherapy regimen changes or the transition between types of treatment were regarded as a treatment line transition. The percentage on the label covers the proportion of the number of patients to all patients in the identical line of trajectory. As the large number of nodes hinders the purpose of the visualizations within a graphical summary, the nodes are truncated at the third node. For the same reason, the nodes for patient count under 10 were removed. AUSOM: Ajou University School of Medicine; RT: radiation therapy.

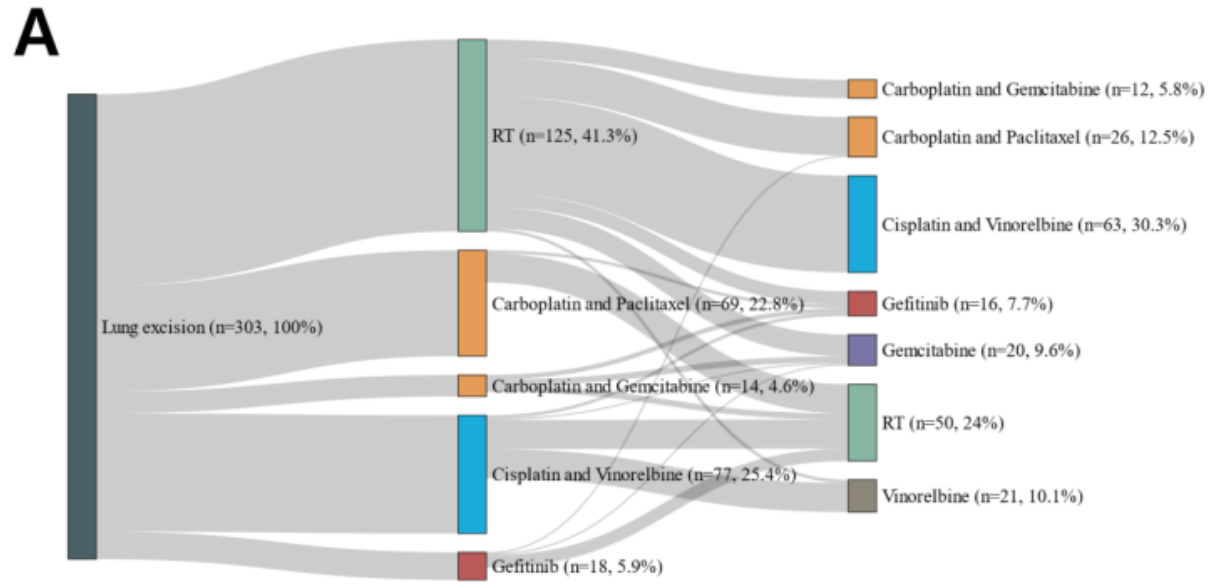

B
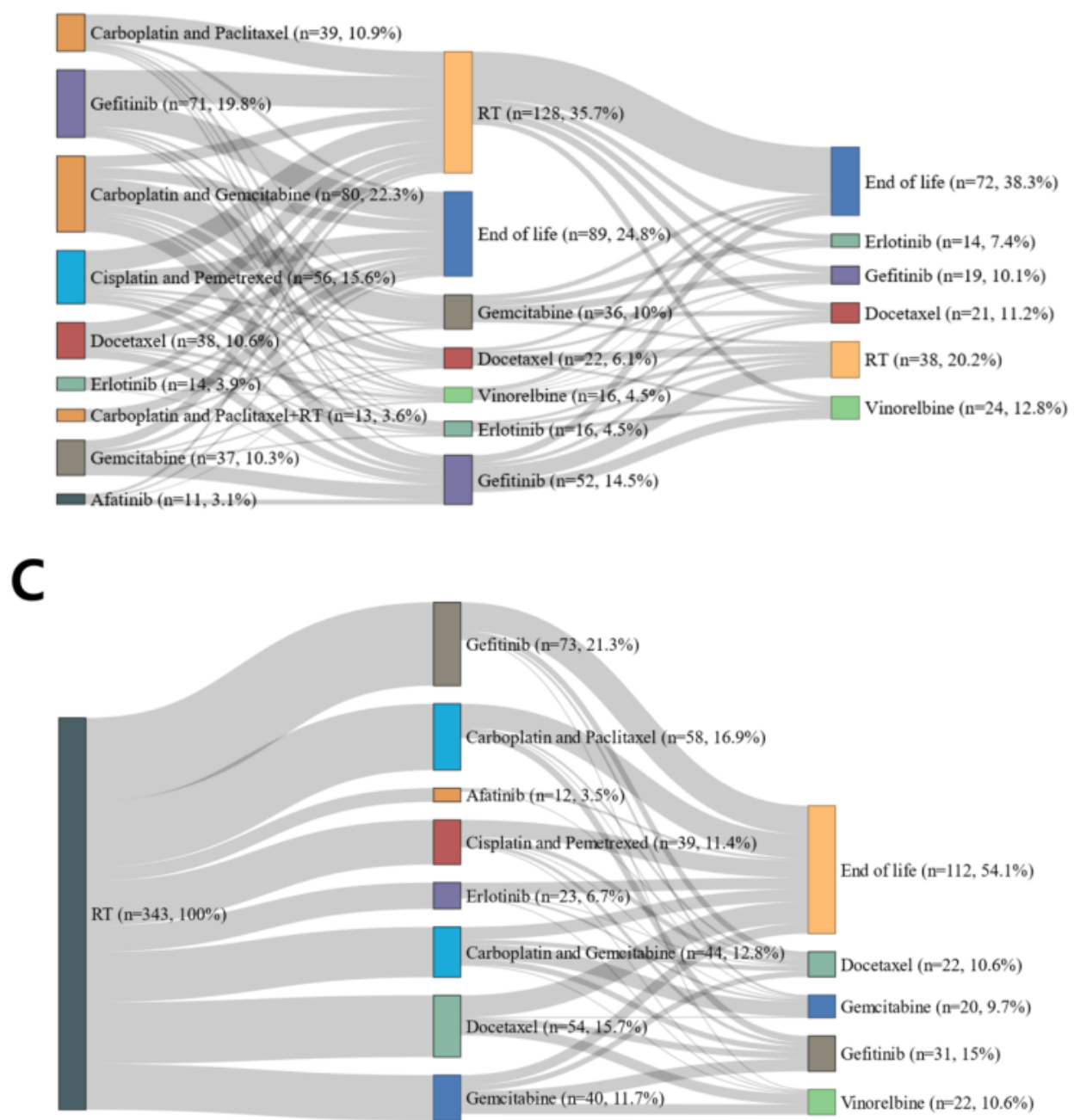
The treatment trajectories of patients in the $\mathrm{KDH}$ database are illustrated in Multimedia Appendix 7. Among the patients with colorectal cancer, FOLFOX was the most frequently used first-line $(n=52,17.9 \%)$ and second-line $(n=84,28.9 \%)$ regimen in the trajectory. For breast cancer, Taxotere was the most frequent first-line chemotherapy before mastectomy $(n=58$, $17.3 \%)$. Gefitinib was the most widely used first-line regimen among patients with lung cancer $(n=30,10.5 \%)$. Figure 6 shows the treatment trajectories of patients with malignant neoplasm who also had COVID-19. Of the 7590 patients nationwide with a diagnosis of COVID-19, we identified 382 patients with a history of cancer. Among them, a total of 62 patients had an episode of chemotherapy. Most of the patients received only one line of treatment between 2017-2020, before COVID-19 infection $(n=47)$. The trajectory included 6 patients with a node of end of life.

Figure 6. Anticancer treatment trajectories of patients with COVID-19. Sankey plot of the treatment trajectories of patients with COVID-19, including episodes of anticancer chemotherapy between 2017 and 2020. Each node represents the chemotherapy regimen used for cancer treatment. The percentage on the label covers the proportion of the patient number in each node in the same trajectory phase. As the large number of nodes hinders the purpose of the visualizations within a graphical summary, the nodes are truncated at the fourth node. For the same reason, the nodes for patient count $<5$ were removed.

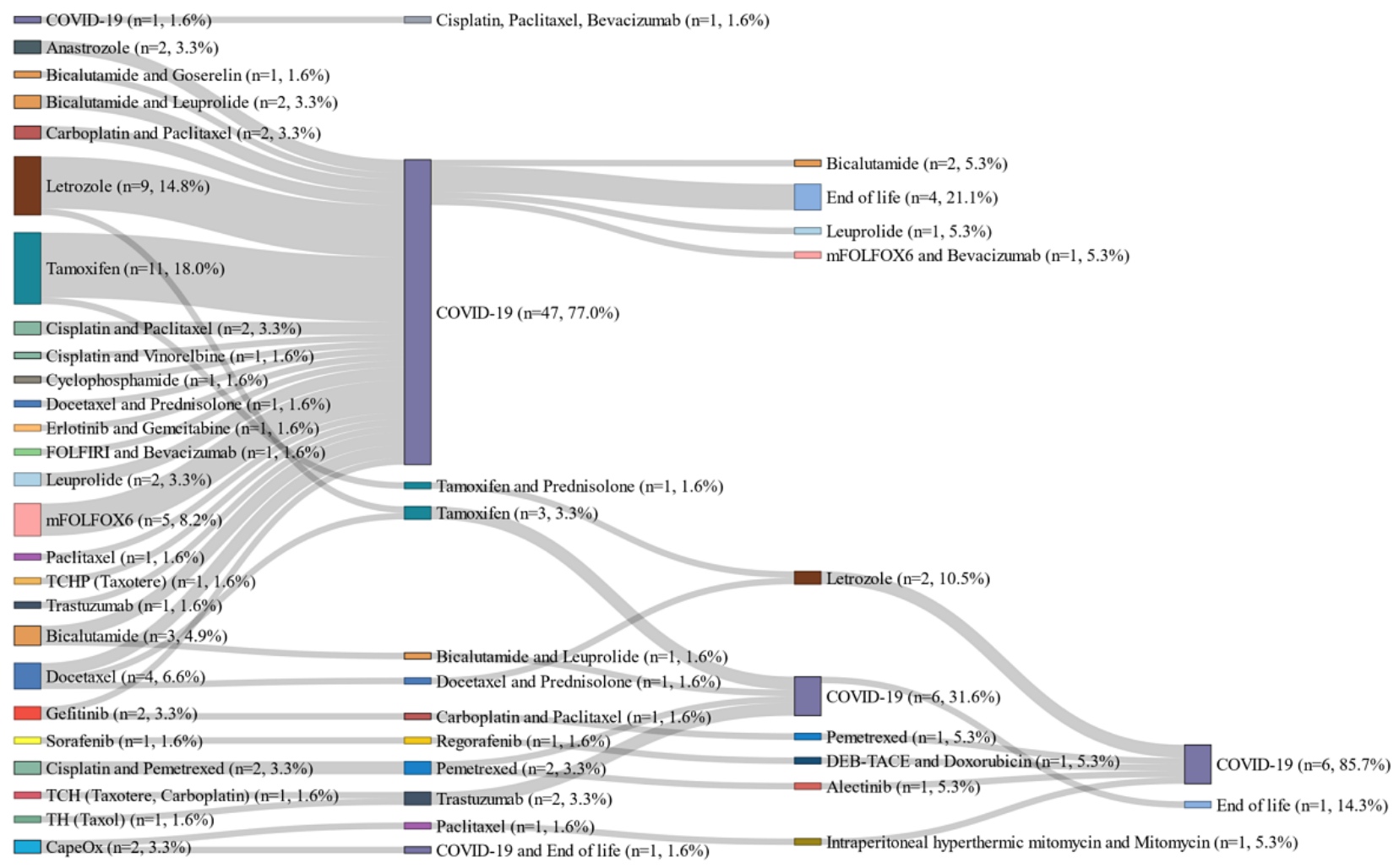

\section{Timing of Chemotherapy-Induced Neutropenia}

Figure 7 shows the time of onset of the CIN/FN event for each patient in the AUSOM database. The episodes of neutropenia among patients with colorectal cancer were clustered between days 9 and 15. Compared with the regimens used for colorectal cancer, the neutropenia events that were recorded after docetaxel monotherapy and Taxotere treatment for breast cancer and after carboplatin and gemcitabine for lung cancer generally began one week earlier (days 2-8). We illustrated the incidence of neutropenia events in each cycle of treatment in Multimedia Appendix 8. Regardless of the cancer type, the incidence of CIN/FN events was high during the first cycle, with the exception of the FOLFOX regimen for colorectal cancer and the carboplatin and paclitaxel regimen for lung cancer. Finally, neutropenia occurred more frequently during the Taxotere regimen for breast cancer (75.3\%), which includes doxorubicin and docetaxel as constituent drugs. 
Figure 7. Trends in neutropenia onset time according to regimen. Time of onset of chemotherapy-induced (febrile) neutropenia event after the first exposure to chemotherapy among patients with (A) breast cancer, (B) colorectal cancer, and (C) lung cancer at the Ajou University School of Medicine. Each dot represents the neutropenia event of a distinct patient. The events are categorized in a 7-day range. The violin plot represents the trends in the frequency on each day from chemotherapy exposure. AC: doxorubicin and cyclophosphamide; FAC: fluorouracil, doxorubicin, and cyclophosphamide; FOLFIRI: fluorouracil, leucovorin, and irinotecan; FOLFOX: fluorouracil, leucovorin, and oxaliplatin.

A

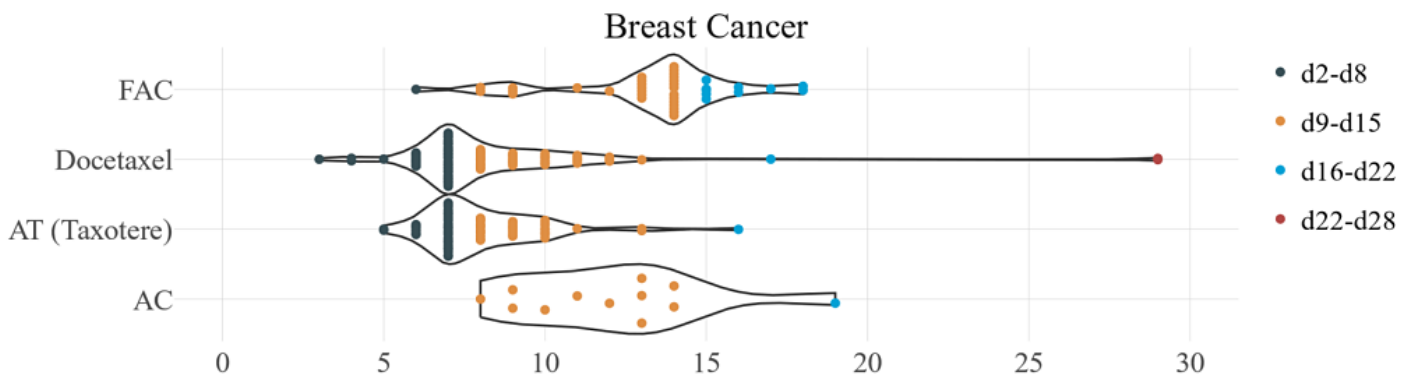

B

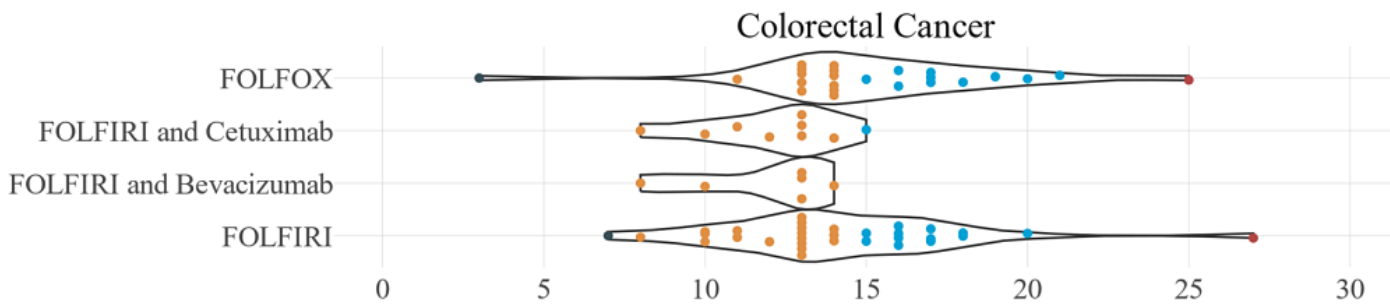

C

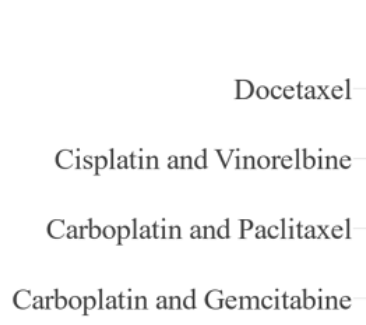

Lung Cancer

\section{Discussion}

\section{Overview}

This study described a system for analyzing the treatment patterns and trajectories of patients with cancer based on the oncology extension model in the OMOP-CDM. The proposed algorithm (TRACER) for extracting chemotherapy episodes at the regimen level effectively generated the treatment episodes for patients with cancer. This approach illustrates how laborious manual curation can be replaced with an automatic extraction system. The obtained episodes were validated by reviewing clinical notes, which revealed that the type of regimen or the number of treatment cycles were estimated with high accuracy. We also demonstrated the usefulness of the proposed system by performing a pilot study investigating the onset time of CIN/FN across various chemotherapy regimens.

\section{Principal Findings}

Comprehensive clinical information, including longitudinal treatment sequences and the various clinical outcomes of patients with cancer, is not available in nationwide cancer registries, such as the Surveillance, Epidemiology, and End Results Program [16-18] or the Korea Central Cancer Registry
[19,20]. Large-scale real-world data derived from EHRs [21] and administrative claims data [17] of a standardized data network can support the timely assessment of the characterization and quality of routine clinical practice and active pharmacovigilance across institutions or countries.

The unexpectedly rapid spread of COVID-19 revealed an urgent unmet need for the timely retrieval of detailed data for patients with cancer, to provide relevant evidence for the management of patients with cancer during the pandemic period [22]. Although conventional cancer registries have failed to provide these data to researchers, the secondary use of EHRs and claims databases can promptly provide valuable insights into the impact of a novel infection on patients with cancer [13]. The TRACER was able to generate records to describe the trajectory of cancer treatment and death of patients with COVID-19, which may be helpful for identifying the relationship between cancer treatment and a fatal case of COVID-19.

We demonstrated how electronically captured data elements can support clinical research using longitudinal detailed clinical data. FN is one of the most common oncologic emergencies [23] and is associated with considerable morbidity and mortality [24]. Although it is well known that the risk of CIN/FN is highest during the first cycle of chemotherapy for solid tumors 
or lymphoma [25,26], the exact time of occurrence of CIN/FN in various regimens is largely unknown. We found that CIN/FN events were more frequent in the first cycle of chemotherapy, and that regimens that included docetaxel or doxorubicin were followed by a greater number of CIN/FN events, which is compatible with reported findings [27]. CIN/FN events usually occurred relatively early (days 2-8) in patients who received regimens including docetaxel or carboplatin compared with those treated with other regimens (days 7-13).

\section{Limitations}

This study had several limitations. First, only four of the regimens were validated through manual review; thus, it was not clear whether episodes for the other types of regimen can also be precisely estimated. Nevertheless, the patterns of treatment cycle repetition showed that the extracted records were concordant with the standard protocols of the regimens, suggesting that the algorithm could correctly interpret the variable regimens. A second limitation was that the relatively low rate of ascertained treatment episodes (maximum of 58\% among patients with breast cancer in the AUSOM database) suggests that treatment episodes may have been missed, although many patients with cancer are treated with surgery or radiation alone and would have appropriately not been captured. This may be due to the fact that the algorithm extracts only the regimens included in the HemOnc vocabulary. The flexible structure of HDAC, which allows the addition of user-defined rules for specific regimens, has the potential to mitigate the missing rate of treatment episodes for a particular study using a fine-tuned algorithm.

\section{Conclusions}

We developed a technique to generate episodes for chemotherapies included in the oncology module of the OMOP-CDM and to analyze treatment patterns in patients with cancer. We demonstrated that the proposed process is reproducible and scalable across a distributed data network. Our findings suggest that a generalizable strategy of characterizing treatment trajectories from harmonized observational databases can promptly determine the characteristics of clinical events, thus enabling the generation of real-world evidence for abrupt pandemic crises. Further research is required to generate statistical evidence for clinical outcomes between regimen types.

\section{Acknowledgments}

SCY, HJ, and RWP contributed to the study design. All authors contributed to the writing and final approval of this manuscript. This work was supported by the Bio Industrial Strategic Technology Development Program (20001234) funded by the Ministry of Trade, Industry \& Energy (MOTIE, Korea) and a grant from the Korea Health Technology R\&D Project through the Korea Health Industry Development Institute (KHIDI), funded by the Ministry of Health \& Welfare, Republic of Korea (grant number HI16C0992). JLW was supported by the National Cancer Institute (grant number CA231840).

\section{Conflicts of Interest}

JW has equity in HemOnc.org LLC; this equity has no financial value. JW is the chief software architect of the HemOnc ontology; this role is uncompensated. The development of HemOnc is partially supported by NIH grants U24 CA194215; U01 CA231840; and U24 CA248010. The funder had no role in the development or conduct of the study.

\section{Multimedia Appendix 1}

Chemotherapy episodes from the Ajou University School of Medicine database. The 10 most frequently used chemotherapy regimens for colorectal cancer, breast cancer, and lung cancer are listed.

[DOCX File, 23 KB-Multimedia Appendix 1]

\section{Multimedia Appendix 2}

Trends of chemotherapy regimen use within the Ajou University School of Medicine database. The proportions of chemotherapy regimen uses for patients with (A) colorectal cancer, (B) breast cancer, and (C) lung cancer by year from 2008-2018 in the Ajou University School of Medicine database are shown.

[DOCX File, 492 KB-Multimedia Appendix 2]

\section{Multimedia Appendix 3}

Trends of chemotherapy regimen use within the Kangdong Sacred Heart Hospital database. The proportions of chemotherapy regimen uses for patients with (A) colorectal cancer, (B) breast cancer, and (C) lung cancer by year from 2008-2018 in the Kangdong Sacred Heart Hospital database are shown.

[DOCX File, 167 KB-Multimedia Appendix 3] 


\section{Multimedia Appendix 4}

Heat map of patient distribution for cycle iteration by regimen type in the Kangdong Sacred Heart Hospital database. The number of patients with (A) colorectal cancer, (B) breast cancer, and (C) lung cancer is shown; treatment iteration counts are represented by a color saturation difference.

[DOCX File, 837 KB-Multimedia Appendix 4]

\section{Multimedia Appendix 5}

Trends of chemotherapy regimen use from the Kangdong Sacred Heart Hospital database. The proportions of chemotherapy regimen uses for patients with (A) colorectal cancer, (B) breast cancer, and (C) lung cancer by year from 2008-2018 in the Kangdong Sacred Heart Hospital database are shown.

[DOCX File, $561 \mathrm{~KB}$-Multimedia Appendix 5]

\section{Multimedia Appendix 6}

The list of treatment trajectories of patients with cancer from the Ajou University School of Medicine database.

[DOCX File, $21 \mathrm{~KB}-$ Multimedia Appendix 6]

\section{Multimedia Appendix 7}

Anticancer treatment trajectories for patients with cancer in the Kangdong Sacred Heart Hospital database. The treatment trajectories of patients with (A) colorectal cancer, (B) breast cancer, and (C) lung cancer in the Kangdong Sacred Heart Hospital database are shown.

[DOCX File, 605 KB-Multimedia Appendix 7]

\section{Multimedia Appendix 8}

Incidence of neutropenia by treatment cycle. The histogram of incidence of the first neutropenia event by cycle and regimen for (A) colorectal cancer, (B) lung cancer, and (C) breast cancer.

[DOCX File, 165 KB-Multimedia Appendix 8]

\section{References}

1. Khozin S, Blumenthal GM, Pazdur R. Real-world Data for Clinical Evidence Generation in Oncology. J Natl Cancer Inst 2017 Nov 01;109(11). [doi: 10.1093/jnci/djx187] [Medline: 29059439]

2. Bendell JC, Bekaii-Saab TS, Cohn AL, Hurwitz HI, Kozloff M, Tezcan H, et al. Treatment patterns and clinical outcomes in patients with metastatic colorectal cancer initially treated with FOLFOX-bevacizumab or FOLFIRI-bevacizumab: results from ARIES, a bevacizumab observational cohort study. Oncologist 2012;17(12):1486-1495 [FREE Full text] [doi: 10.1634/theoncologist.2012-0190] [Medline: 23015662]

3. Dasari A, Bergsland EK, Benson AB, Cai B, Huynh L, Totev T, et al. Treatment Patterns and Clinical Outcomes in Advanced Lung Neuroendocrine Tumors in Real-World Settings: A Multicenter Retrospective Chart Review Study. Oncologist 2019 Aug;24(8):1066-1075 [FREE Full text] [doi: 10.1634/theoncologist.2018-0520] [Medline: $\underline{30610008}$ ]

4. Nadler E, Espirito JL, Pavilack M, Boyd M, Vergara-Silva A, Fernandes A. Treatment Patterns and Clinical Outcomes Among Metastatic Non-Small-Cell Lung Cancer Patients Treated in the Community Practice Setting. Clin Lung Cancer 2018 Jul;19(4):360-370 [FREE Full text] [doi: 10.1016/j.cllc.2018.02.002] [Medline: 29576407]

5. Bikov KA, Mullins CD, Seal B, Onukwugha E, Hanna N. Algorithm for identifying chemotherapy/biological regimens for metastatic colon cancer in SEER-Medicare. Med Care 2015 Aug;53(8):e58-e64. [doi: 10.1097/MLR.0b013e31828fad9f] [Medline: 23552436]

6. Carroll NM, Burniece KM, Holzman J, McQuillan DB, Plata A, Ritzwoller DP. Algorithm to Identify Systemic Cancer Therapy Treatment Using Structured Electronic Data. JCO Clin Cancer Inform 2017 Nov; 1:1-9 [FREE Full text] [doi: 10.1200/CCI.17.00002] [Medline: 30657379]

7. Prodel M, Lamarsalle L, Augusto V. ATLAS: a robust algorithm for temporal sequence alignment of treatment lines using claim databases. 2019 Presented at: 2019 IEEE Conference on Computational Intelligence in Bioinformatics and Computational Biology (CIBCB); July 9-11, 2019; Siena, Italy p. 1-8. [doi: 10.1109/cibcb.2019.8791467]

8. Weymann D, Costa S, Regier DA. Validation of a Cyclic Algorithm to Proxy Number of Lines of Systemic Cancer Therapy Using Administrative Data. JCO Clin Cancer Inform 2019 Aug;3:1-10 [FREE Full text] [doi: 10.1200/CCI.19.00022] [Medline: $\underline{31365273}$ ]

9. Hripcsak G, Duke JD, Shah NH, Reich CG, Huser V, Schuemie MJ, et al. Observational Health Data Sciences and Informatics (OHDSI): Opportunities for Observational Researchers. Stud Health Technol Inform 2015;216:574-578 [FREE Full text] [Medline: 26262116] 
10. Belenkaya R, Gurley M, Dymshyts D, Araujo S, Williams A, Chen R, et al. Standardized Observational Cancer Research Using the OMOP CDM Oncology Module. Stud Health Technol Inform 2019 Aug 21;264:1831-1832. [doi: 10.3233/SHTI190670] [Medline: $\underline{31438365]}$

11. Warner JL, Dymshyts D, Reich CG, Gurley MJ, Hochheiser H, Moldwin ZH, et al. HemOnc: A new standard vocabulary for chemotherapy regimen representation in the OMOP common data model. J Biomed Inform 2019 Aug;96:103239 [FREE

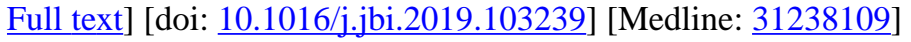

12. Yoon D, Ahn EK, Park MY, Cho SY, Ryan P, Schuemie MJ, et al. Conversion and Data Quality Assessment of Electronic Health Record Data at a Korean Tertiary Teaching Hospital to a Common Data Model for Distributed Network Research. Healthc Inform Res 2016 Jan;22(1):54-58 [FREE Full text] [doi: 10.4258/hir.2016.22.1.54] [Medline: 26893951]

13. Burn E, You SC, Sena AG, Kostka K, Abedtash H, Abrahão MTF, et al. An international characterisation of patients hospitalised with COVID-19 and a comparison with those previously hospitalised with influenza. medRxiv. Preprint published online on April 25, 2020 [FREE Full text] [doi: 10.1101/2020.04.22.20074336] [Medline: $\underline{32511443}$ ]

14. Warner JL, Cowan AJ, Hall AC, Yang PC. HemOnc.org: A Collaborative Online Knowledge Platform for Oncology Professionals. J Oncol Pract 2015 May;11(3):e336-e350 [FREE Full text] [doi: 10.1200/JOP.2014.001511] [Medline: 25736385]

15. Jeon HK, You SC. CancerTxPathway. GitHub. 2020. URL: https://github.com/ABMI/CancerTxPathway [accessed 2020-10-15]

16. Bach PB, Guadagnoli E, Schrag D, Schussler N, Warren JL. Patient demographic and socioeconomic characteristics in the SEER-Medicare database applications and limitations. Med Care 2002 Aug;40(8 Suppl):IV-19. [doi: 10.1097/00005650-200208001-00003] [Medline: 12187164]

17. Enewold L, Parsons H, Zhao L, Bott D, Rivera DR, Barrett MJ, et al. Updated Overview of the SEER-Medicare Data: Enhanced Content and Applications. J Natl Cancer Inst Monogr 2020 May 01;2020(55):3-13. [doi:

10.1093/jncimonographs/lgz029] [Medline: 32412076]

18. Warren JL, Klabunde CN, Schrag D, Bach PB, Riley GF. Overview of the SEER-Medicare data: content, research applications, and generalizability to the United States elderly population. Med Care 2002 Aug;40(8 Suppl):IV-I3. [doi: 10.1097/01.MLR.0000020942.47004.03] [Medline: 12187163]

19. Jung K, Won Y, Kong H, Lee ES. Cancer Statistics in Korea: Incidence, Mortality, Survival, and Prevalence in 2016. Cancer Res Treat 2019 Apr;51(2):417-430 [FREE Full text] [doi: 10.4143/crt.2019.138] [Medline: 30913865]

20. Shin H, Won Y, Jung K, Kong H, Yim S, Lee J, Members of the Regional Cancer Registries. Nationwide cancer incidence in Korea, 1999 2001; first result using the national cancer incidence database. Cancer Res Treat 2005 Dec;37(6):325-331 [FREE Full text] [doi: 10.4143/crt.2005.37.6.325] [Medline: 19956367]

21. Ma X, Long L, Moon S, Adamson B, Baxi S. Comparison of Population Characteristics in Real-World Clinical Oncology Databases in the US: Flatiron Health, SEER, and NPCR. MedRxiv. Preprint published online on May 30, 2020. [doi: 10.1101/2020.03.16.20037143]

22. Kuderer NM, Choueiri TK, Shah DP, Shyr Y, Rubinstein SM, Rivera DR, COVID-19 Cancer Consortium. Clinical impact of COVID-19 on patients with cancer (CCC19): a cohort study. Lancet 2020 Jun 20;395(10241):1907-1918 [FREE Full text] [doi: 10.1016/S0140-6736(20)31187-9] [Medline: 32473681]

23. Lewis MA, Hendrickson AW, Moynihan TJ. Oncologic emergencies: Pathophysiology, presentation, diagnosis, and treatment. CA Cancer J Clin 2011;61(5):287-314 [FREE Full text] [doi: 10.3322/caac.20124] [Medline: 21858793]

24. Kuderer NM, Dale DC, Crawford J, Cosler LE, Lyman GH. Mortality, morbidity, and cost associated with febrile neutropenia in adult cancer patients. Cancer 2006 May 15;106(10):2258-2266 [FREE Full text] [doi: 10.1002/cncr.21847] [Medline: 16575919]

25. Culakova E, Thota R, Poniewierski MS, Kuderer NM, Wogu AF, Dale DC, et al. Patterns of chemotherapy-associated toxicity and supportive care in US oncology practice: a nationwide prospective cohort study. Cancer Med 2014 Apr;3(2):434-444 [FREE Full text] [doi: 10.1002/cam4.200] [Medline: 24706592]

26. Crawford J, Dale DC, Kuderer NM, Culakova E, Poniewierski MS, Wolff D, et al. Risk and timing of neutropenic events in adult cancer patients receiving chemotherapy: the results of a prospective nationwide study of oncology practice. J Natl Compr Canc Netw 2008 Feb;6(2):109-118. [doi: 10.6004/jnccn.2008.0012] [Medline: 18319047]

27. Lalami Y, Paesmans M, Muanza F, Barette M, Plehiers B, Dubreucq L, et al. Can we predict the duration of chemotherapy-induced neutropenia in febrile neutropenic patients, focusing on regimen-specific risk factors? A retrospective analysis. Ann Oncol 2006 Mar;17(3):507-514 [FREE Full text] [doi: 10.1093/annonc/mdj092] [Medline: 16322116]

\section{Abbreviations}

AC: cyclophosphamide and doxorubicin

ANC: absolute neutrophil count

AUSOM: Ajou University School of Medicine

CapeOx: capecitabine and oxaliplatin

CDM: common data model 
CIN/FN: chemotherapy-induced (febrile) neutropenia

CMF: cyclophosphamide, methotrexate, and fluorouracil

CRCAE: Common Terminology Criteria for Adverse Events

EHR: electronic health record

FAC: fluorouracil, doxorubicin, and cyclophosphamide

FEC: fluorouracil, epirubicin, and cyclophosphamide

FOLFIRI: fluorouracil, leucovorin, and irinotecan

FOLFOX: fluorouracil, leucovorin, and oxaliplatin

FULV: fluorouracil and folinic acid

HDAC: Hierarchical Description for Administration of Chemotherapy

HIRA: Health Insurance Review and Assessment Service

JSON: JavaScript Object Notation

KDH: Kangdong Sacred Heart Hospital

OHDSI: Observational Health Data Sciences and Informatics

OMOP: Observational Medical Outcomes Partnership

TRACER: Tool for Regimen-level Abstraction of Chemotherapy Episode Records

Edited by G Eysenbach; submitted 15.10.20; peer-reviewed by J Banda, L Rusu; comments to author 07.11.20; revised version received 12.11.20; accepted 20.01.21; published 06.04.21

Please cite as:

Jeon H, You SC, Kang SY, Seo SI, Warner JL, Belenkaya R, Park RW

Characterizing the Anticancer Treatment Trajectory and Pattern in Patients Receiving Chemotherapy for Cancer Using Harmonized Observational Databases: Retrospective Study

JMIR Med Inform 2021;9(4):e25035

URL: https://medinform.jmir.org/2021/4/e25035

doi: $10.2196 / 25035$

PMID: $\underline{3720842}$

(CHokyun Jeon, Seng Chan You, Seok Yun Kang, Seung In Seo, Jeremy L Warner, Rimma Belenkaya, Rae Woong Park. Originally published in JMIR Medical Informatics (http://medinform.jmir.org), 06.04.2021. This is an open-access article distributed under the terms of the Creative Commons Attribution License (https://creativecommons.org/licenses/by/4.0/), which permits unrestricted use, distribution, and reproduction in any medium, provided the original work, first published in JMIR Medical Informatics, is properly cited. The complete bibliographic information, a link to the original publication on http://medinform.jmir.org/, as well as this copyright and license information must be included. 